\title{
THE ROAD TO DIPLOID ANDROGENESIS
}

\author{
(The Japan Society of Human Genetics Award Lecture)
}

Tadashi KAJII

Department of Pediatrics, Yamaguchi University School of Medicine, Ube 755, Japan

\section{INTRODUCTION}

In the aftermath of the publication of androgenetic origin of complete hydatidiform moles (Kajii and Ohama, 1977), I was frequently asked if the discovery was made overnight by a flip of the fingers. It was not, but it took several agonizing years with several ups and downs. All the while, I kept saying that I knew the answer. I did not. By telling the story, I would reveal that I was an idiot. Nevertheless, I am tempted to do so, partly because this is a fitting occasion to tell such a story. No space is allotted for such an episode in a serious scientific article. It is not to say that giving a lecture upon receiving this prestigious award is not serious business, but the audience would be broad-minded enough to allow me to indulge myself in reminiscences. This way, I would be able to convey the circumstances in which the studies were made that eventually led to the discovery of androgenetic origin of complete moles.

\section{GYNOGENESIS VS ANDROGENESIS}

My interest in hydatidiform moles was first aroused in early 1960s by watching direct squash chromosome slides prepared by then young Drs. Motomichi Sasaki, Masao S. Sasaki and Tsutomu Fukushima at Prof. Makino's laboratory in Sapporo, a five minutes' walk from my laboratory. The slides were prepared by mincing grape-like vesicles picked up from a bunch immersed in the sea of blood (Fig. 1). The quality of the chromosomes so prepared was not as good as that one would expect in today's preparations. Yet, it was possible to tell that the great majority, if not all, of hydatidiform moles are 46,XX (Sasaki et al., 1962; Makino et al., 1965). 


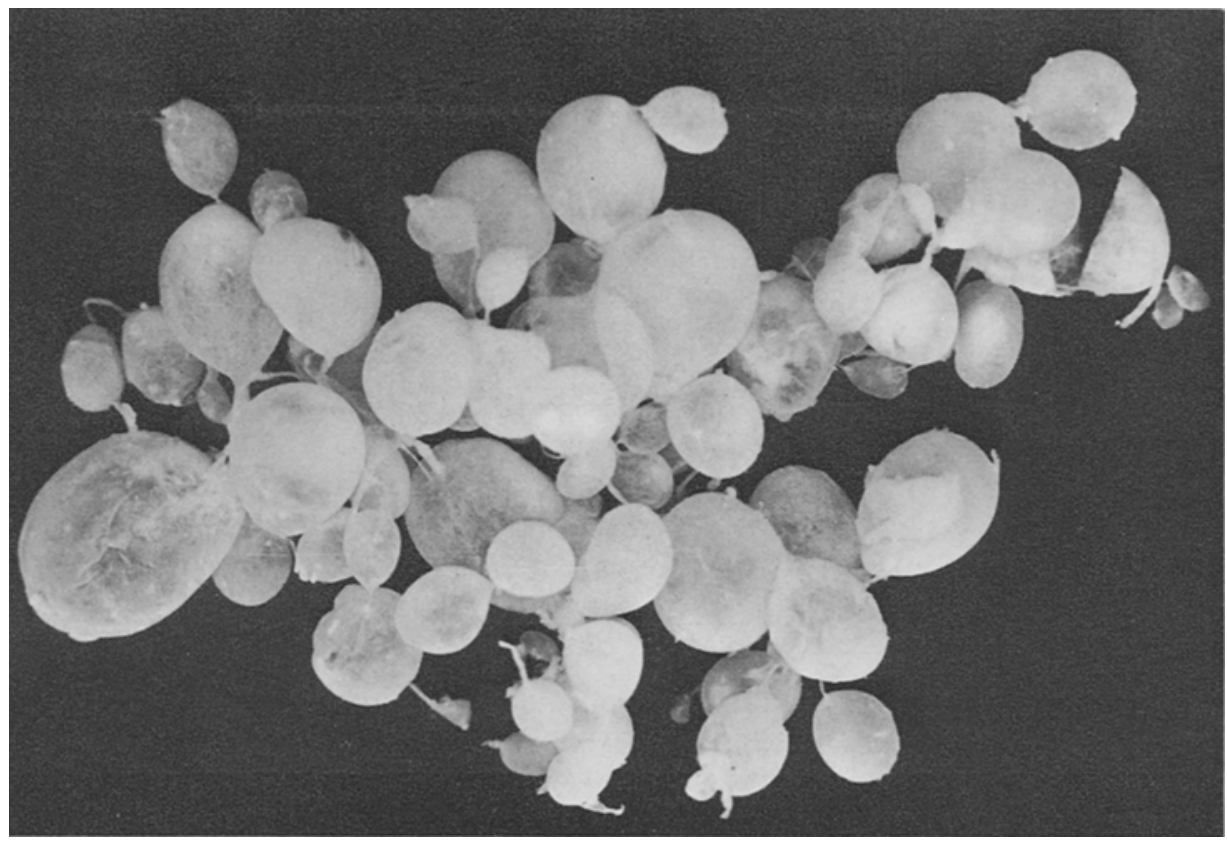

Fig. 1. Complete mole $(46, \mathrm{XX})$ with swollen villi.

This finding was also supported by X-chromatin studies and karyotyping cultured molar fibroblasts by others (World Health Organization, 1966). Prof. Makino and his colleagues also reported on three triploid specimens with an unusual villous pattern, partial moles in today's classification (Makino et al., 1964).

Later in Geneva, when engaged in cytogenetic studies in spontaneous abortuses, we occasionally encountered molar specimens. Geneva was not the place appropriate for the study of complete moles, because molar pregnancies are infrequent in Europe and North America, occurring at a rate of one in 1,000 to 2,000 births. Molar pregnancies are much more frequent in Asia including Japan, where they are encountered at a rate of one in 100-500 pregnancies. In the meantime, I attended a lecture in Geneva given by Prof. Hertig, an authority on histology of trophoblastic diseases. His position was that hydatid chorionic villi progress with progressing pregnancy to partial mole and then to complete mole (Hertig, 1968). This to me was not acceptable because partial moles are mostly triploid while complete moles are diploid. Thus, the time was ripe to think about the peculiar dominance of female sex in complete moles.

Several possibilities were conceivable to account for the predominantly or exclusively female sex in complete moles (Table 1). 1) It could be that moles have a $1: 1$ sex ratio at conception, but $\mathrm{XY}$ moles are somehow eliminated during early development, thus leaving only XX moles. 2) Duplication of the $23, X$ second polar 
Table 1. Possible origins of $46, \mathrm{XX}$ complete moles.
1. Selection against $46, \mathrm{XY}$ moles
2. Duplication of the second polar body
3. Diploid parthenogenesis
4. Diploid androgenesis
5. Diploid gynogenesis

body of an ovum was proposed by Baggish et al. (1968). Under this scheme, the female and male pronuclei in the ovum conceived develop to become an XX or $\mathrm{XY}$ fetus, while the second polar body duplicates itself to become an XX mole. The rapidly proliferating mole in most instances would overgrow the fetus. Rarely both lines might develop, resulting in the occurrence of a twin pregnancy consisting of a fetus and a mole. 3) Diploid parthenogenesis, i.e. duplication of the female pronucleus in an ovum, would result in a 46,XX karyotype. This, however, can not explain why moles occur exclusively in married or sexually active women. 4) Diploid androgenesis, i.e. exclusion of the female pronucleus of an ovum and duplication of the male pronucleus, would result in either an $\mathrm{XX}$ or YY diploid conceptuses. The $Y Y$ conceptuses are lethal in the absence of the $X$ chromosome. This mechanism fits with the facts that moles occur in sexually active women and that they are apparently exclusively female. We figured that diploid androgenesis is unlikely to be the mechanism leading to complete moles, mainly because it involves two unlikely events, elimination or inactivation of the female pronucleus and duplication of the male pronucleus. 5) Thus, gynogenesis, a variation of parthenogenesis, seemed to be the most likely mechanism. Gynogenesis, unlike parthenogenesis, requires penetration of an ovum by a sperm. The penetrated sperm activates the ovum, but the sperm itself fails to participate in subsequent development and thus degenerates. The activated egg refrains from emitting a polar body and so becomes diploid without a contribution from the sperm. This would explain both the apparently exclusive female sex of complete moles and their occurrence among sexually active women. A unique mole with a 48,XXYY karyotype described by Shinohara et al. of Tokyo (1971) seemed to indicate doubling of a genome. The supposed YY chromosomes could have been GG chromosomes. Doubling of a $24, \mathrm{X},+\mathrm{G}$ gamete would result in a $48, \mathrm{XX},+\mathrm{G},+\mathrm{G}$ karyotype.

Gynogenesis is a normal and only means of reproduction of a fish called Amazon molly (Poecilia formosa). The species apparently arose as an interspecific hybrid between $P$. latipinna and $P$. mexicana and are all females. Amazon mollies mate with a male sailfin molly, $P$. latipinna, one of the parental species. The male's sperm does not contribute genetically to the genome of $P$. formosa, but his sperm merely serves to induce parthenogenetic development in the diploid egg of the Amazon molly.

It was 1972. We were then culturing and karyotyping spontaneous abortuses, and were trying to develop a method to determine the parental origin of chromosome 
abnormalities in spontaneous abortions. Eventually, a sequential Q- and R-banding method was developed (Niikawa and Kajii, 1975). Its application to triploid abortuses revealed the majority to have resulted from dispermy (Kajii and Niikawa,
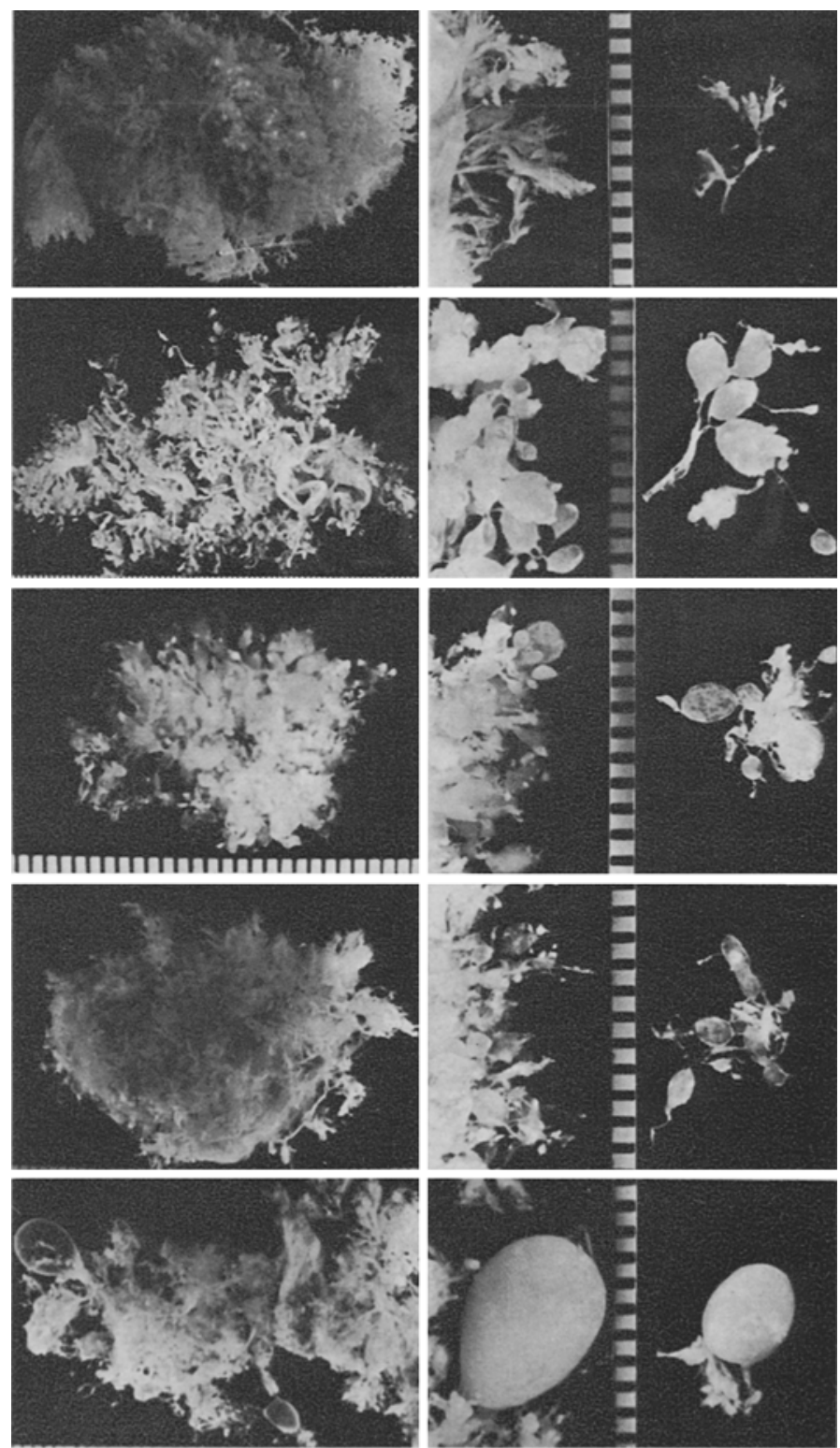

Fig. 2. Normal villi in normal placenta (first row) and various types of partial mole (second through bottom rows). 
1977). The same trick would work on complete moles. We knew from our experience that culturing chorionic villi is a risky business. When kept more than a week in culture, the villous cells are taken over by the surrounding decidual cells. Chromosomes from the molar cells, being gynogenetic in origin, would be indistinguishable from decidual cells which are of maternal origin. Thus, we resorted to uncultured, direct chromosome preparation from molar trophoblasts. We also tried not to mix true, complete moles with partial moles. Partial moles, unlike complete moles, consisted of various chromosome abnormalities including triploidy (Vassilakos et al., 1977). It was possible to distinguish complete from partial moles by checking them under an inverted microscope (Fig. 2).

We made Q- and R-banding analyses of chromosome preparations from uncultured molar specimens and from cultured peripheral blood lymphocytes of the parents. While the Q- and R-banding patterns of direct preparations of molar chromosomes were of a miserable quality, we repeatedly saw homozygous banding patterns on homologous molar chromosomes. This further fed our conviction that moles are gynogenetic in origin, but their quality was not good enough to prove that. In the meantime, our colleague, Dr. Ohama, left our laboratory and started to work in Hiroshima. He prepared chromosome slides from moles in Hiroshima and sent them to Geneva.

We suffered for three years from second-rate chromosomes. We felt beaten when Linder $e t$ al. (1975) described in early 1975 on parthenogenetic origin of benign ovarian teratoma, because we then believed that we were striving for the same goal (Fig. 3). Dr. Niikawa, our colleague, then working on Q- and R-banding, twice raised the possibility that moles are androgenetic. While they were difficult to photograph, the fluorescent polymorphic patterns of molar homologous chromosomes, when observed under a microscope, resembled those of a paternal chromosome. Each time I rejected the suggestion, saying that it is impossible for two unlikely events to occur at the same time at the same place.

In early 1976, a mole aborted by a Spanish woman yielded direct chromosome slides of excellent quality. At about the same time, Dr. Ohama, being less inhibited than the author, cultured a molar specimen from a Japanese woman. In both, the banding patterns of informative homologous chromosomes were homozygous. There was no maternal contribution, while in each informative chromosomal pair a paternal chromosome was transmitted in duplicate to the mole (Fig. 3). We deduced that a functionally anucleate ovum (with its nucleus either inactivated or eliminated with a polar body) was fertilized by either a haploid sperm and then it duplicated itself to restore diploidy, or by a diploid sperm resulting from non-division at the second meiotic division (Fig. 4), a process called diploid androgenesis. Our a priori reasoning, gynogenesis, was proven wrong. Because preconception did not enter the picture, the observation must have been correct.

Nevertheless, we kept silence for a year, piling up more evidence. In the meantime, Wake and Takagi in Prof. M. Sasaki's laboratory in Sapporo made a 


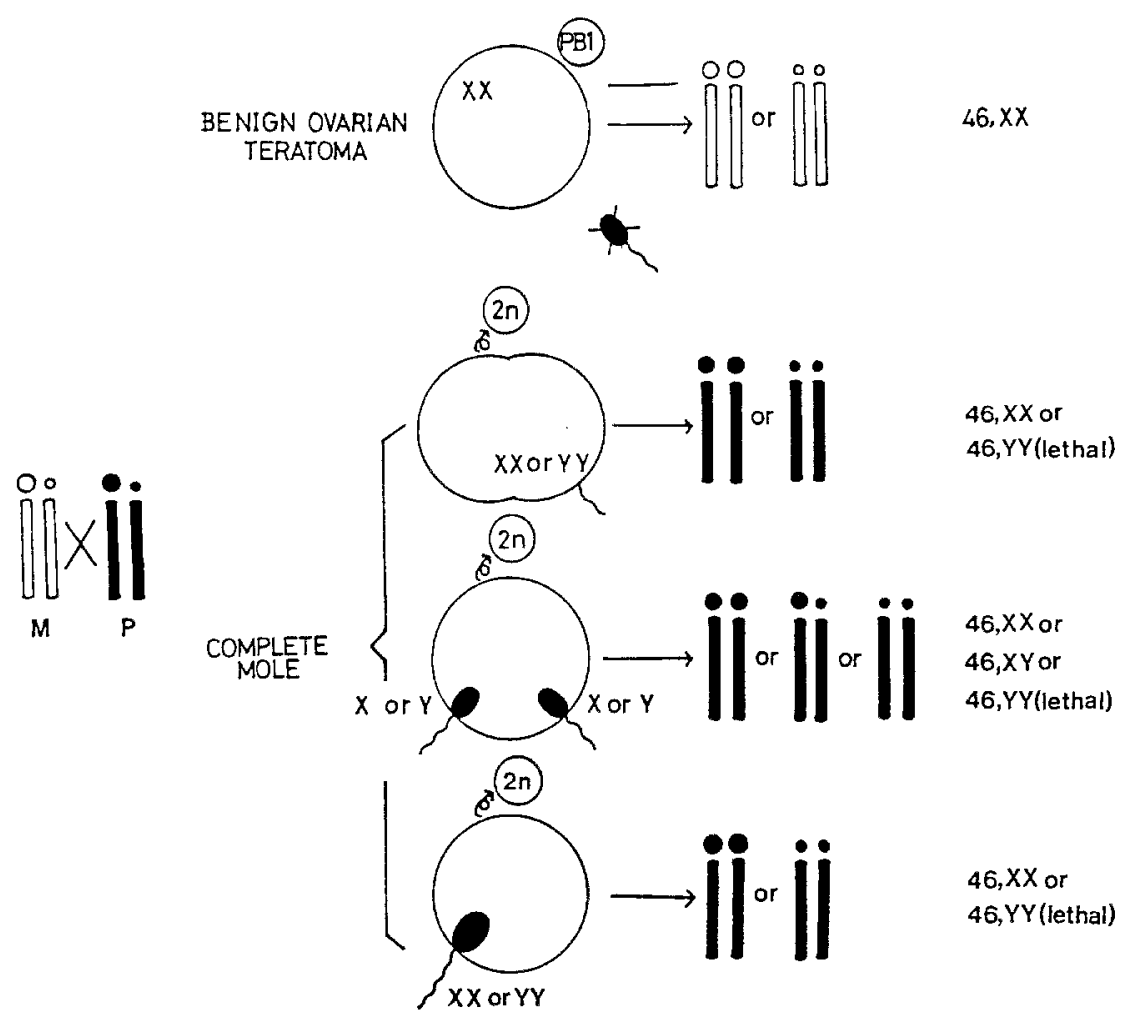

Fig. 3. The diagram illustrates the conceivable modes of origin of benign ovarian teratoma and complete mole. The latter includes duplication of a haploid sperm (top), dispermy (middle) and diplospermy (bottom).

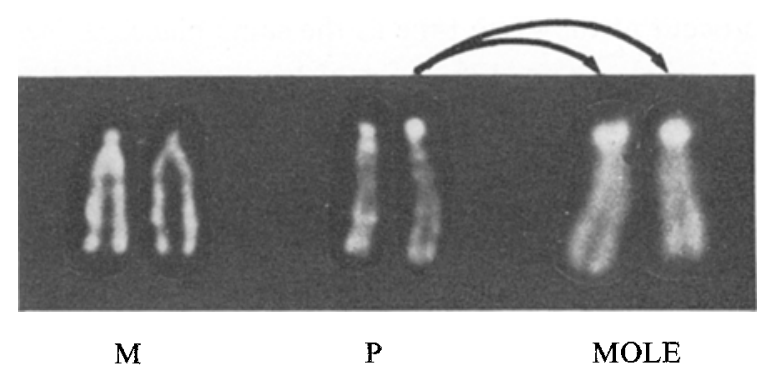

Fig. 4. R banded chromosomes 14 from a complete mole (MOLE), its host (M) and her husband (P). A paternal chromosome 14 was transmitted to the mole in duplicate.

similar study and obtained the same result. Prof. Sasaki, being aware that we had a big fish, asked in his letter whether the fish was an androgenone. This was the last kick we needed. We rushed for publication. The paper published in August 1977 included seven XX moles, two from Caucasian and five from Japanese women, all 


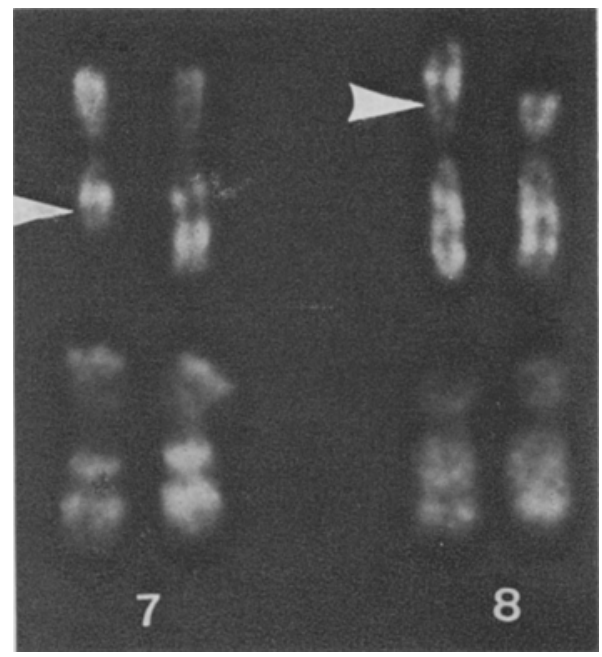

Fig. 5. A $t(7 ; 8)(q 22 ; p 21)$ translocation in a woman who had a molar pregnancy. $\mathrm{Q}$ banding. Maternal (top) and molar (bottom) chromosomes.

of which were of androgenetic origin (Kajii and Ohama, 1977). The paper also included two women with a molar pregnancy each of whom had a balanced reciprocal translocation. One of them, $t(7 ; 8)(\mathrm{q} 22 ; \mathrm{p} 21)$, is shown in the accompanying figure (Fig. 5). The presence of translocation chromosomes may interfere with female meiosis and encourage the production of ova with absent or inactivated nuclei. The paper by Wake et al. was published in 1978 and essentially supported our findings (Wake et al., 1978). Their paper also proved that one of the two X chromosomes in molar cells was late-replicating. Inactivation of the $X$ chromosome may occur in the absence of the maternally-derived haploid set.

\section{LATER DEVELOPMENTS}

Many findings have since been made. Androgenetic origin of complete moles was supported by various studies that employed not only chromosomal heteromorphisms but also HLA polymorphisms, isozymes and restriction fragment length polymorphisms (Yamashita et al., 1979; Jacobs et al., 1980; Lawler et al., 1982; Hoshina et al., 1984). Complete, homozygous moles were proven to originate from duplication of a haploid sperm that entered an ovum, not from the conception by a diploid sperm resulting from nondivision at second meiosis (Jacobs et al., 1980). This was concluded in view of the absence of heterozygous segments distal to the sites of meiotic crossing over (Fig. 6). According to the latter scheme, the recombinant segments would contain heterozygous loci for which the father is heterozygous.

A second variety of moles, usually with a $46, X Y$ karyotype and with a less than 


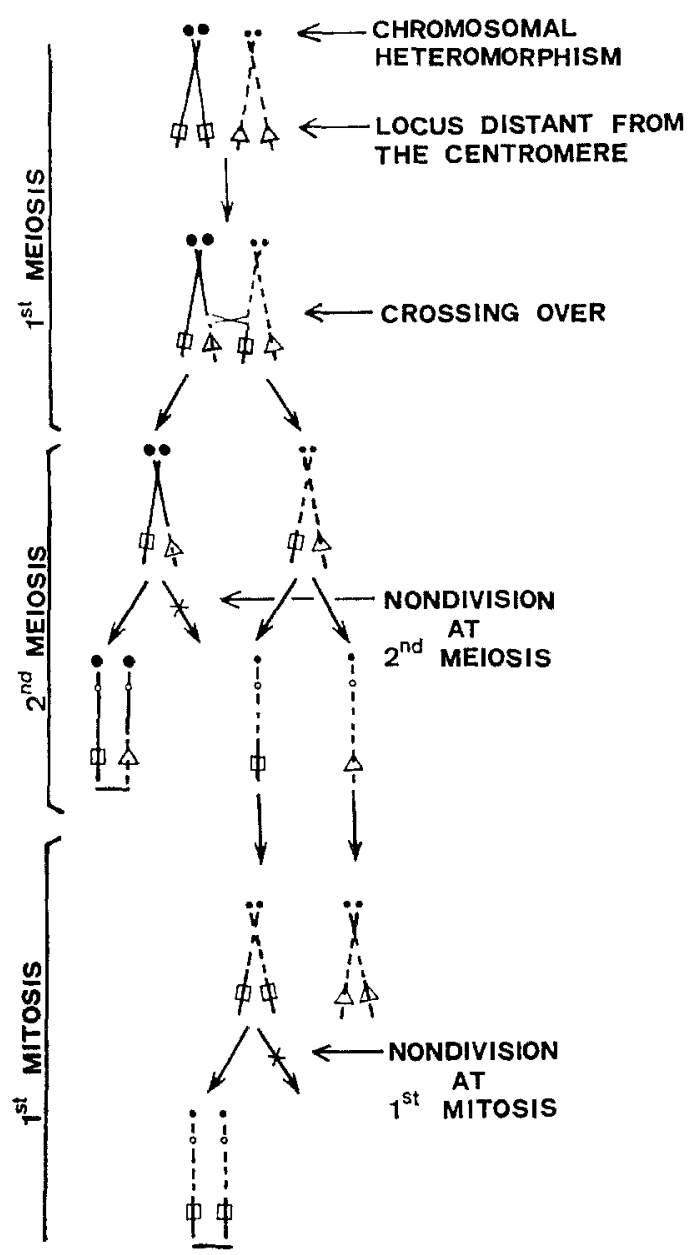

Fig. 6. Simplified presentation of the difference between nondivision at second meiosis (left) and that at first mitosis (right), showing behavior of a pair of homologous chromosomes with heterozygosity at a locus close to the centromere (chromosomal heteromorphism) and a locus distant from it. Crossing-over has occurred between the centromere and the distant locus. Nondivision at second meiosis leads to heterozygosity of the locus, while that at first mitosis leads to its homozygosity. In both, the chromosomal marker, close to the centromere, loses its heterozygosity.

$10 \%$ frequency of the entire molar population, was proven to result from dispermy (Ohama et al., 1981). The dispermic moles are 50\% homozygous and 50\% heterozygous for the loci for which the father is heterozygous. Moles from diplospermy (fertilization by a diploid sperm) may exist, but even if they existed, they are so rare that they would be negligible (Fig. 3). XY, heterozygous moles are grossly and histologically indistinguishable from XX, homozygous moles (Kajii et al., 1984). 
The DNA replication patterns of $\mathrm{X}$ chromosomes of molar fibroblasts are similar to those of adult and fetal fibroblasts, but different from those in adult lymphocytes, indicating that DNA replication kinetics of molar fibroblasts are tissue-specific rather than origin or developmental-stage specific (Tsukahara and Kajii, 1985).

Studies on mitochondrial DNA polymorphisms in complete moles indicated that molar mitochondrial DNA is of maternal origin while the nuclear chromosomes are entirely paternal (Wallace et al., 1982; Edwards et al., 1984). This further supported the interpretation that moles arise from the fusion of one or two sperm with a mature but anucleate ovum.

Combined cytogenetic and histologic studies have shown that in triploid partial moles the extra haploid set is almost always of paternal origin and that in non-molar triploids the extra set is of maternal origin (Jacobs et al., 1982). Tetraploid partial moles with a $92, \mathrm{XXXY}$ karyotype were proven to have resulted from trispermy (Surti et al., 1986). These findings, together with those in complete moles, indicate that the formation of swollen villi is associated with an excess of the paternal over the maternal haploid sets. Evidence in support of this conclusion came from experimental studies on mice in which activated parthenogenetic or fertilized anucleate eggs were variously reconstituted with exogenous male and female pronuclei. Gynogenetic embryos, containing two female pronuclei, developed abnormally and characteristically produced a poor trophoblast while the embryo proper developed to some degree (Surani and Barton, 1983). On the other hand, mouse eggs containing two male pronuclei, a situation equivalent to a complete mole, showed a markedly better development, while the embryo itself died early (Barton et al., 1984). Such findings imply that the maternal genome is essential for embryogenesis, while the paternal genome is important in the establishment of the extra-embryonic tissues, especially the trophoblast. These findings led to the hypothesis that the maternal and paternal genomes undergo a separate specific imprinting during oogenesis and spermatogenesis, respectively, and that the maternal and paternal genomic contributions are not equivalent (McGrath and Solter, 1984; Surani et al., 1984). The paternally derived $\mathrm{X}$ chromosome was predominantly inactivated in certain extraembryonic tissues of developing female murine embryos (Takagi and Sasaki, 1975). In female murine embryos of parthenogenetic origin, the frequency of cells with a late replicating $\mathrm{X}$ was significantly lower in the extraembryonic region than in that of normally fertilized embryos (Endo and Takagi, 1981).

Many puzzles pertaining to complete moles remain to be solved. Is complete homozygosity as observed in XX moles and in some ovarian teratomas at a disadvantage by comparison with heterozygosity in terms of malignant transformation (Kajii, 1980; Fisher and Lawler, 1984; Wake et al., 1984; Ohama et al., 1985)? How does a woman, with a molar pregnancy and challenged by two sets of paternal genes, suppress the activity of the maternal immune system to permit the pregnancy to continue? Further studies of complete moles will provide answers to these and other questions. 
Acknowledgment I would like to express my gratitude to my colleagues Drs. $\mathrm{K}$. Ohama, P. Vassilakos, N. Niikawa, Ariane Ferrier and Ms. Elizabeth Merotto for their collaboration, to Prof. K. Mikamo for introducing me into human embryology, and to the gynecologists who supplied molar specimens. Our works quoted in this paper were supported by grants-in-aid from the Ford Foundation, the World Health Organization, the Swiss National Foundation, the Ministry of Education, Science and Culture of Japan and the Princess Takamatsu Cancer Research Foundation.

\section{REFERENCES}

Baggish, M.S., Woodruff, J.D., Tow, S.H., and Jones, H.W., Jr. 1968. Sex chromatin pattern in hydatidiform mole. Am. J. Obstet. Gynecol. 102: 362-370.

Barton, S.C., Surani, M.A.H., and Norris, M.L. 1984. Role of paternal and maternal genomes in mouse development. Nature 311: 374-376

Edwards, Y.H., Jeremiah, S.J., McMillan, S.L., and Povey, S. 1984. Complete hydatidiform moles combine maternal mitochondria with a paternal nuclear genome. Ann. Hum. Genet. 48: 119127.

Endo, S. and Takagi, N. 1981. A preliminary cytogenetic study of X chromosome inactivation in diploid parthenogenetic embryos from LT/Sv mice. Jpn. J. Human Genet. 56: 349-356.

Fisher, R.A. and Lawler, S.D. 1984. Heterozygous complete hydatidiform moles: Do they have a worse prognosis than homozygous complete moles? Lancet ii: 51 only.

Hertig, A.T. 1968. Human Trophoblast. Charles C. Thomas. Springfield, U.S.A.

Hoshina, M., Boothby, M.R., Hussa, R.D., Pattillo, R.A., Camel, H.M., and Boime, I. 1984. Segregation patterns of polymorphic restriction sites of the gene encoding the $\alpha$ subunit of human chorionic gonadotropin in trophoblastic disease. Proc. Natl. Acad. Sci. U.S.A. 81: 2504-2507.

Jacobs, P.A., Wilson, C.M., Sprenkle, J.A., Rosenshein, N.B., and Migeon, B.R. 1980. Mechanism of origin of complete hydatidiform moles. Nature 286: 714-716.

Jacobs, P.A., Szulman, A.F., Funkhouser, J., Matsuura, J.S., and Wilson, C.C. 1982. Human triploidy: relationship between parental origin of the additional haploid complement and development of partial hydatidiform mole. Ann. Hum. Genet. 46: 223-231.

Kajii, T. and Niikawa, N. 1977. Origin of triploidy and tetraploidy in man: 11 cases with chromosome markers. Cytogenet. Cell Genet. 18: 109-125.

Kajii, T. and Ohama, K. 1977. Androgenetic origin of hydatidiform mole. Nature 268: 633-634.

Kajii, T. 1980. Androgenetic origin of hydatidiform moles: Its bearing on carcinogenesis. Gann Monogr. Cancer Res. 25: 189-194.

Kajii, T., Kurashige, H., Ohama, K., and Uchino, F. 1984. XY and XX complete moles: Clinical and morphologic correlations. Am. J. Obstet. Gynecol. 150: 57-64

Lawler, S.D., Povey, S., Fisher, R.A., and Pickthall, V.J. 1982. Genetic studies on hydatidiform moles. II. The origin of complete moles. Ann. Hum. Genet. 46: 209-222.

Linder, D., Kaiser, McCaw, B., and Hecht, F. 1975. Parthenogenetic origin of benign ovarian teratomas. New Engl. J. Med. 292: 63-66.

Makino, S., Sasaki, M.S., and Fukuschima, T. 1964. Triploid chromosome constitution in human chorionic lesions. Lancet ii: 1273-1275.

Makino, S., Sasaki, M.S., and Fukuschima, T. 1965. Cytological studies of tumors. XLI. Chromosomal instability in human chorionic lesions. Okajima Folia Anat. Jpn. 40: 439-465.

McGrath, J. and Solter, D. 1984. Completion of mouse embryogenesis requires both maternal and paternal genomes. Cell 137: 179-183.

Niikawa, N. and Kajii, T. 1975. Sequential Q- and acridine orange-marker technique. Humangenetik 30: 83-90.

Ohama, K., Kajii, T., Okamoto, E., Fukuda, Y., Imaizumi, K., Tsukahara, M., Kobayashi, K., and Hagiwara, K. 1981. Dispermic origin of XY hydatidiform moles. Nature 292: 551-552. 
Ohama, K., Nomura, K., Okamoto, E., Fukuda, Y., Ihara, T., and Fujiwara, A. 1985. Origin of immature teratoma of the ovary. Am. J. Obstet. Gynecol. 152: 896-900.

Sasaki, M., Fukuschima, T., and Makino, S. 1962. Some aspects of the chromosome constitution of hydatidiform moles and normal chorionic villi. Gann 53: 101-106.

Shinohara, T., Sasaki, M.S., Tonomura, A., Shimamine, T., Yokoyama, T., and Hasegawa, T. 1971. Cytogenetic studies in human chorionic lesions. Jpn. J. Human Genet. 16: 111-112.

Surani, M.A.H. and Barton, S.C. 1983. Development of gynogenetic eggs in the mouse: implications for parthenogenetic embryos. Science 222: 1034-1036.

Surani, M.A.H., Barton, S.C., and Norris, M.L. 1984. Development of reconstituted mouse eggs suggests imprinting of the genome during gametogenesis. Nature 308: 548-550.

Surti, U., Szulman, A.E., Wagner, K., Leppert, M., and O'Brien, S.J. 1986. Tetraploid partial hydatidiform moles: two cases with a triple paternal contribution and a 92,XXXY karyotype. Hum. Genet. 72: 15-21.

Takagi, N. and Sasaki, M. 1975. Preferential inactivation of the paternally derived X chromosome in the extraembryonic membranes of the mouse. Nature 256: 640-642.

Tsukahara, M. and Kajii, T. 1985. Replication of X chromosomes in complete moles. Hum. Genet. 71: 7-10.

Vassilakos, P., Riotton, G., and Kajii, T. 1977. Hydatidiform mole: Two entities. A morphologic and cytogenetic study with some clinical considerations. Am. J. Obstet. Gynecol. 127: 167-170.

Wake, N., Takagi, N., and Sasaki, M. 1978. Androgenesis as a cause of hydatidiform mole. $J$. Natl. Cancer Inst. 60; 51-57.

Wake, N., Seki, T., Fujita, H., Ohkubo, H., Sakai, K., Okuyama, K., Hayashi, H., Shiina, Y., Sato, H., Kuroda, M., and Ichinoe, K. 1984. Malignant potential of homozygous and heterozygous complete moles. Cancer Res. 44: 1226-1230.

Wallace, D.C., Surti, U., Adams, C.W., and Szulman, A.E. 1982. Complete moles have partial chromosomes but maternal mitochondrial DNA. Hum. Genet. 61: 145-147.

World Health Organization. 1966. Standardization of procedures for chromosome studies in abortion. Bull. Wld. Health Org. 34: 765-782.

Yamashita, K., Wake, N., Araki, T., Ichinoe, K., and Kuroda, M. 1979. Human lymphocyte antigen expression in hydatidiform mole: Androgenesis following fertilization by a haploid sperm. Am. J. Obstet. Gynecol. 135: 597-600. 